\title{
Producción científica de las universidades mexicanas en el área de la Cultura Física: una revisión sistematizada \\ Scientific production of Mexican universities in Physical Culture: a systematized review \\ Ramón Candia Lujan, Lidia Guillermina De León Fierro, Claudia Esther Carrasco Legleu, Briseidy Ortiz Rodriguez, Kevin \\ Fernado Candia Sosa, Julio César Guedea Delgado \\ Universidad Autónoma de Chihuahua (México)
}

\begin{abstract}
Resumen. El objetivo del presente estudio fue analizar la producción científica en el área de la Cultura Física publicada por parte de las universidades públicas mexicanas. Se llevó a cabo una revisión en la base de datos Scopus. Del total de universidades públicas estatales mexicanas, 20 cuentan alguna licenciatura relacionada con la cultura física. La búsqueda se limitó a artículos, revisiones y artículos en prensa de 1990 a 2018. Se encontró que la producción total de documentos fue de 258, en 48 hubo colaboración nacional y en 156 internacional. En la mayoría de los documentos un investigador de estas instituciones fue el autor principal y/o de correspondencia del documento. De forma particular sobresalen la Universidad Autónoma de Chihuahua, la Autónoma de Nuevo León y la de Guadalajara como las principales productoras en el área. Se concluye que durante estos 28 años la producción científica en el área es escasa.
\end{abstract}

Palabras clave: Artículos, Revisiones, Producción Científica, Universidades Mexicanas.

\begin{abstract}
The aim of the present study was to analyze the scientific production in the area of Physical Culture published by Mexican public universities. A search was carried out in the Scopus database. Of the total of Mexican state public universities, 20 offer degrees related to physical culture. The review was limited to articles, reviews, and press articles from 1990 to 2018 . The total of produced documents was 258, 48 implying national collaboration whereas 156 international. In most of the documents, an investigator from these institutions was the main and / or correspondent author of the document. Particularly noteworthy are the Autonomous University of Chihuahua, the Autonomous University of Nuevo León, and Guadalajara University as the main producers in the area. As a conclusion, during these 28 years, scientific production in the area have been scarce.
\end{abstract}

Keywords: Articles, Reviews, Scientific Production, Mexican Universities.

\section{Introducción}

La producción científica se refiere a todos aquellos documentos en los que se encuentran registradas y difundidas las investigaciones de un área de la ciencia (De Jesus Nascimento, 2016) siendo uno de sus principales criterios de productividad, la cantidad de artículos publicados (Reverter Masià, Hernández González, Jové Deltell \& Legaz Arrese, 2013).

La autoevaluación de la productividad científica de los investigadores y de las universidades mismas es un ejercicio necesario, ya que regularmente la productividad representa un parámetro de calidad por el que se reciben beneficios y apoyos de instancias gubernamentales (HernándezGonzález, Reverter-Masià \& Jové-Deltell, 2016).

La investigación es considerada una función sustantiva de las instituciones de educación superior (IES) y en los últimos años se ha considerado que la calidad de las universidades tiene una estrecha relación con la producción científica (Piedra-Salomón \& Martínez-Rodríguez, 2007) a la par de la docencia y la extensión de la cultura, todas ellas, actividades fundamentales de estas instituciones (Arechavala, 2011).

Una de las estrategias para impulsar la producción científica en México fue la creación del Sistema Nacional de Investigadores (SNI) en 1984, dependiente del Consejo Nacional de Ciencia y Tecnología (CONACyT), con el objetivo de reconocer a los profesionales dedicados a la investigación científica y tecnológica. Para ser parte del SNI el aspirante solicita la evaluación del número y calidad de sus productos

Fecha recepción: 08-10-19. Fecha de aceptación: 27-02-20 Julio César Guedea Delgado

jcguedea@uach.mx científicos a través de una revisión por pares académicos (Acosta-Ramírez, Ordaz-Cortes \& Baquero-Parra, 2015).

Doce años más tarde, en 1996, la Secretaría de Educación Pública estableció el Programa del Mejoramiento del Profesorado (PromeP), actualmente Programa para el Desarrollo Profesional Docente del Tipo Superior (PRODEP), con el objetivo de impulsar la formación de alto nivel de los profesores de las IES del país (Maranto-Rivera, 2017). El programa establece la figura del Perfil Deseable PRODEP, el cual es otorgado a profesores que realizan equilibradamente las funciones de docencia, investigación, tutoría y gestión académica (De Vries, González, León, \& Hernández, 2008).

En México, las áreas de biología y química, ciencias sociales, físico-matemáticas y ciencias de la tierra, han sido las más frecuentemente elegidas por la mayoría de los investigadores adscritos a universidades públicas o centros de investigación (Cabrero-Mendoza, 2015). Por su parte, los estudios en las ciencias de la cultura física, educación física o bien actividad física y deporte, han tenido un importante crecimiento en las tres décadas recientes. Si bien en países como Brasil (de Souza-Méndez, et al., 2017), Chile (PérezGutiérrez, Lagos-Hernández, \& Izquierdo-Macón, 2106) y España (Devis-Devis, Valenciano, Villamón \& PérezSamaniego, 2010; Reverter-Masià, Hernández-González, JovéDeltell, \& Legaz-Arrese, 2016) se han llevado a cabo estudios donde se ha evaluado la trayectoria de la producción científica en esta área, en México no existe algún estudio al respecto.

El objetivo del presente trabajo fue analizar la producción científica en el área de la cultura física, que se desarrolla en las universidades públicas estatales mexicanas, que se encuentran incluidas en la base de datos Scopus. 
Universidades públicas estatales con programas de licenciatura y/o posgrado, relacionados a las Ciencias de la Cultura Física.

\begin{tabular}{|c|c|c|c|}
\hline Universidad & Dirección de página web & Licenciatura & Posgrado \\
\hline A. de Aguascalientes (UAA) & http://www.uaa.mx & Ciencias de la Cultura Física & No \\
\hline A. de Baja California (UABC) & http://www.uabc.mx & Actividad Física y Deporte & Educación Física y Deporte Escolar (M) * \\
\hline A. del Carmen (UNACAR) & http://www.unacar.mx & Educación Física y Deporte & No \\
\hline de Colima (UCOL) & https://www.ucol.mx & Educación Física y Deporte & No \\
\hline A. de Chihuahua (UACH) & http://www.uach.mx & $\begin{array}{l}\text { Educación Física } \\
\text { Motricidad Humana }\end{array}$ & $\begin{array}{l}\text { Ciencias del Deporte (M) } \\
\text { Psicomotricidad (M) } \\
\text { Atención a Poblaciones Especiales (M) } \\
\text { Administración de la Educación Física el Deporte y la Recreación (M) } \\
\text { Ciencias de la Cultura Física* (D) }\end{array}$ \\
\hline A. de Ciudad Juárez (UACJ) & http://www.uacj.mx/paginas/default.aspx & Entrenamiento Deportivo & No \\
\hline Juárez del Estado de Durango (UJED) & https://Www.ujed.mx & Educación Física y Deporte & Cultura Física y Deporte (M) \\
\hline de Guanajuato (UGTO) & http://www.ugto.mx & Ciencias de la Actividad Física y Salud & No \\
\hline de Guadalajara (UDEG) & http://www.udg.mx & Cultura Física & Educación Física y Deporte (M) \\
\hline A. del Estado de México (UAEM) & http://www.uaemex.mx & Cultura Física y Deporte & No \\
\hline A. del Estado de Morelos (UAEMOR) & https://www.uaem.mx & Ciencias Aplicadas al deporte & Ciencias del Deporte $(\mathrm{M})$ \\
\hline A. de Nayarit (UAN) & http://www.uan.edu.mx & Cultura Física y Deporte & - \\
\hline A. de Nuevo León (UANL) & http://www.uanl.mx & Ciencias del Ejercicio & $\begin{array}{l}\text { Actividad Física y Deporte* (M) } \\
\text { Psicología del Deporte* (M) } \\
\text { Ciencias de la Cultura Física } *(\mathrm{D})\end{array}$ \\
\hline A. "Benito Juárez" de Oaxaca (UABJO) & http://www.uabjo.mx & Entrenamiento Deportivo & No \\
\hline Benemérita A. de puebla (BUAP) & https://www.buap.mx & $\begin{array}{l}\text { Cultura Física } \\
\text { Readaptación Física }\end{array}$ & Educación Física y Deporte Escolar (M) \\
\hline de Querétaro (UAQ) & https://www.uaq.mx & Educación Física y Ciencias del Deporte & Ciencias de Rehabilitación en el Movimiento Humano (M) \\
\hline A. de Sinaloa (UAS) & http://web.uas.edu.mx/web/ & $\begin{array}{l}\text { Cultura Física } \\
\text { Educación Deportiva }\end{array}$ & No \\
\hline de Sonora (UNISON) & http://www.uson.mx & Cultura Física y Deporte & No \\
\hline Instituto Tecnológico de Sonora (ITSON & https://www.itson.mx/paginas/index.aspx & $\begin{array}{l}\text { Ciencias del Ejercicio Físico } \\
\text { Dirección de la Cultura Física y el Deporte }\end{array}$ & No \\
\hline Universidad Veracruzana (UV) & https://www.uv.mx & Educación Física Deporte y Recreación & Ciencias Aplicadas a la Actividad Física (M) \\
\hline
\end{tabular}

\section{Metodología}

El presente estudio es una revisión sistematizada (Codina, 2018). Se llevó a cabo una consulta a la base de datos Scopus del 3 al 25 de julio de 2018. La búsqueda de documentos fue por afiliación. Primero se realizó la consulta de la página web de la Subsecretaría de Educación Superior de la Secretaría de Educación Pública de México para conocer las universidades públicas estatales. Posteriormente se consultó cada página web de las 34 universidades, para identificar aquellas que cuentan con algún programa de licenciatura relacionado con la cultura física y ser incluidas en el estudio. En la tabla 1 se presentan las universidades que tienen algún programa educativo del área de interés, si tienen o no programas de posgrado y sus sitios web. El orden de la revisión de las universidades fue de forma aleatoria. El análisis de la producción científica se limitó a la revisión de artículos, revisiones y artículos en prensa.

\section{Procedimiento}

En la figura 1 se muestra un ejemplo del procedimiento de la selección de los artículos de la primera universidad revisada.

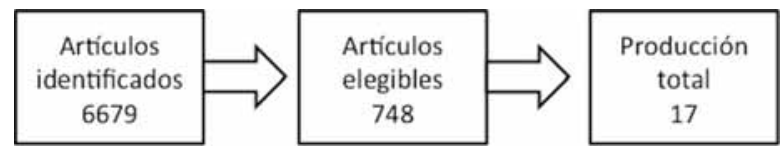

Figura 1. Ejemplo del proceso de revisión y selección final de la producción de la Universidad de Guanajuato

Los artículos incluidos en el presente estudio fueron limitados en años, de 1990 al 2018, en las áreas de medicina y otras profesiones de la salud, ciencias sociales, psicología, y multidisciplinarias; únicamente se consideraron artículos, revisiones y artículos en prensa.

En las universidades que tienen una estructura clásica, por facultades, se incluyeron solo los estudios que estuvieran firmados por algún investigador de la unidad académica que tiene el programa relacionado a las ciencias de la cultura física. Por otra parte, en las universidades con estructura departamental, los artículos seleccionados debían estar firmados por algún investigador que perteneciera a un progra- ma del área de nuestro interés, pero si no informaba el departamento del autor, el trabajo tenía que ser de actividad física, deporte o recreación.

Después de la selección de los artículos por universidad los datos se enviaron a una hoja de Excel para el análisis correspondiente.

\section{Resultados}

En la figura 2 se muestra la secuencia de selección de los artículos identificados. De los 258 artículos finales, en 48 de ellos hubo colaboración nacional y 156 internacional. En 182 artículos el autor principal fue un investigador de las universidades evaluadas y en 187 el autor de correspondencia. El promedio de autores por documento fue de cinco.

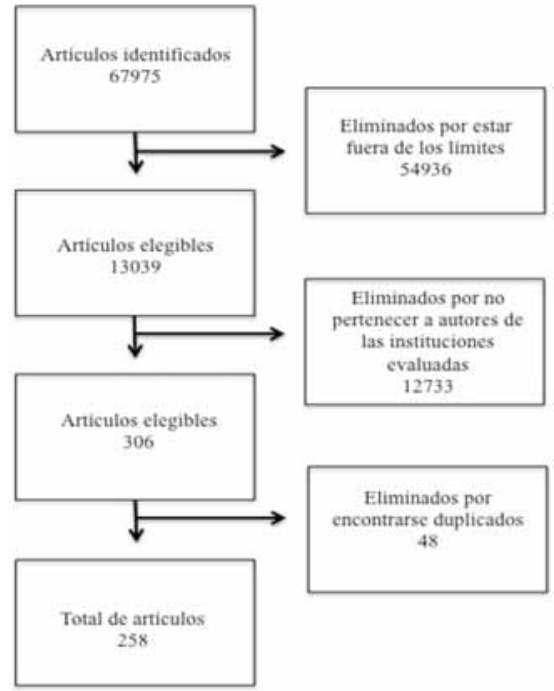

Figura 2. Proceso de selección de la producción científica de la universidades públicas estatales mexicanas.

Las principales revistas en las que publicaron los investigadores fueron: Revista Psicología del Deporte (28 documentos), Nutrición Hospitalaria (28 documentos) y Retos (25 documentos).

En la tabla 2 se muestra la descripción de los artículos encontrados y el orden en que fueron identificados los documentos de cada una de las instituciones 
Tabla 2

Descripción de la producción cientifica de las universidades públicas estatales de México en el área de la cultura física

\begin{tabular}{|c|c|c|c|c|c|c|c|c|c|}
\hline Institución & $\begin{array}{l}\text { Documentos } \\
\text { identificados }\end{array}$ & $\begin{array}{c}\text { Documentos } \\
\text { elegibles }\end{array}$ & $\begin{array}{c}\text { Documentos } \\
\text { de interés }\end{array}$ & Principal revista donde publicaron & $\begin{array}{l}\text { Número de autores } \\
\text { por documento }\end{array}$ & Col nal & Col int & $\begin{array}{c}\text { Autor } \\
\text { principal }\end{array}$ & $\begin{array}{c}\text { Autor de } \\
\text { correspondencia }\end{array}$ \\
\hline UGTO & 6679 & 748 & 17 & Revista de investigación clínica & 5 & 2 & 3 & 15 & 15 \\
\hline UAEMOR & 5448 & 582 & 0 & & & & & & \\
\hline UAQ & 2901 & 496 & 0 & & & & & & \\
\hline UAEM & 3449 & 678 & 13 & Nutrición hospitalaria & 5 & 6 & 6 & 6 & 5 \\
\hline UABC & 4609 & 765 & 26 & Nutrición hospitalaria & 4 & 7 & 15 & 18 & 21 \\
\hline UABJO & 204 & 88 & 0 & & & & & & \\
\hline UJED & 1053 & 332 & 3 & Retos & 4 & 1 & 3 & 3 & 3 \\
\hline ITSON & 531 & 92 & 0 & & & & & & \\
\hline UACJ & 1341 & 290 & 25 & Nutrición hospitalaria & 6 & 22 & 0 & 18 & 18 \\
\hline UAA & 1111 & 247 & 0 & & & & & & \\
\hline UACA & 438 & 23 & 0 & & & & & & \\
\hline UV & 4295 & 1048 & 6 & Revista Iberoamericana de Psicología del ejercicio y el deporte & 6 & 4 & & 3 & 4 \\
\hline UCOL & 2783 & 751 & 6 & Retos & 5 & 1 & 4 & 4 & 3 \\
\hline UNISON & 3776 & 516 & 3 & Nutrición hospitalaria & 4 & 2 & 2 & 2 & 2 \\
\hline UACH & 1320 & 368 & 93 & Formación universitaria & 5 & 36 & 36 & 55 & 55 \\
\hline UAS & 2137 & 365 & 7 & Biomed Research & 6 & 3 & 4 & 0 & 0 \\
\hline BUAP & 9117 & 999 & 4 & Revista Internacional de Medicina y Ciencias de la Actividad Física y el Deporte & 3 & 0 & 0 & 4 & 4 \\
\hline UAN & 641 & 126 & 0 & & & & & & \\
\hline UANL & 6697 & 1504 & 63 & Revista de Psicología del Deporte & 5 & 17 & 53 & 39 & 42 \\
\hline UDEG & 9455 & 3021 & 40 & Retos & 5 & 18 & 30 & 15 & 15 \\
\hline
\end{tabular}

\section{Discusión}

Si bien a través del tiempo la formación del profesorado de educación física ha sido en gran medida dirigido a la aplicación de materiales didácticos y contenidos prefijados por expertos (Moreno-Doña, Arévalo \& Gómez-Gonzalvo, 2020) en los últimos años esta formación ha tomado una orientación más científica (Molina, Martínez-Baena \& Villamon, 2017) por lo que la producción científica en el área ha cobrado gran relevancia.

La cantidad de artículos del área de la cultura física representa menos del $.05 \%$ del total de la producción de las áreas identificadas como frecuentemente elegidas por los investigadores mexicanos, lo que indica que la producción en el campo de interés es pobre en nuestro país. También se considera que la producción es baja si se compara con los países que tienen las universidades más productivas en investigación, como son el caso de las españolas quienes solo en el área de educación física publicaron 1197 artículos y las brasileñas de los que se recopilaron 1581 documentos en la base de datos Scopus (Mariazale, 2011), aunque en el estudio solo se evaluaron los profesores pertenecientes al área y no a las universidades (Reverter-Masià, Hernández-González, Jové-Deltell \& Legaz-Arrese, 2014).

El período a evaluar fue elegido desde 1990 porque coincide con el año de inicio del primer programa de maestría en ciencias en el área en México (Zueck-Enríquez, Chávez-Guerrero \& Blanco-Vega, 2009) y con la exigencia de formación científica de los docentes universitarios (Salazar, 2018), por lo que la formación de científicos se identifica como un objetivo de las universidades, función prioritaria y responsabilidad que recae en el posgrado (Valdez-Ramírez, 2005; Ramírez, et al., 2011). De ahí que en esta revisión se observó que las universidades que mayor producción científica tienen, es porque ofertan algún programa de posgrado, como en el caso de la UACH y la UANL que cuentan con un programa doctoral.

Las revistas científicas son la principal vía y la forma más común para la evaluación de la actividad de los investigadores y desde luego, para la difusión de sus resultados (Villamón-Herrera, Devís-Devís, Valencia-Peris, \& Valenciano-Valcárcel, 2007). De las revistas en español, en las que más publican los investigadores mexicanos en ciencias de la cultura física, la Revista de Psicología del Deporte la cual está directamente relacionada con el área, la Revista Nutri- ción Hospitalaria publica diversos temas, entre los que se encuentran la actividad física y el deporte y Retos, es una revista dedicada específicamente a la educación física. Solo una de las universidades (UAS) evaluadas evidenció como principal revista de publicación una en inglés.

En su estudio Reverter-Masià et al. (2014) encontraron que las tres primeras revistas en Scopus en la que publicaban tanto investigadores españoles como brasileños en el tema de la educación física fue el Journal of Strength and Conditioning Research. En un estudio posterior estos mismos autores (Reverter-Masià et al., 2016) reportaron que los profesores funcionarios españoles con sexenio en esa área, publicaron más en las revistas Archivos de Medicina del Deporte, European Journal Applied Physiology y Journal of Sport Medicine and Physical Fitness. Aunque para los investigadores españoles de las ciencias del deporte las revistas en inglés más valoradas son el Journal of Sport Sciences y Sport Medicine (Ortega \& Salado, 2018).

La colaboración de ámbito nacional o internacional se puede determinar por medio de la coautoría (Alonso-Arroyo, Oliveira, Grácio, Pandiella \& Aleixandre-Benavent, 2016). Es de gran importancia conocer este parámetro. La colaboración tanto a nivel nacional como internacional de los investigadores mexicanos en el área de la cultura física ocurre en aproximadamente la mitad de los artículos publicados en Scopus, por debajo del $63 \%$ de la colaboración que reciben los autores españoles del área de ciencias de la actividad física y el deporte (Valenciano-Valcárcel, Devís-Devís, Villamón \& Peiró-Velert, 2010). En este aspecto, sobresale de forma particular la UACJ en la colaboración nacional y la UANL en la internacional, ya que la mayoría de sus documentos los han publicado junto a investigadores nacionales o internacionales respectivamente.

El autor principal es considerado generalmente, el de mayor importancia ya que, hace la mayor parte del trabajo hasta su redacción o es quien ha desarrollado el objetivo de la investigación (Mattar \& González, 2011) mientras que el autor de correspondencia regularmente se refiere al investigador que fue el autor de la idea principal y ha dirigido la investigación o ambos; es además el que tiene contacto con el editor de la revista durante el proceso de revisión del artículo, por lo que estos dos autores pueden considerarse como los dos más importantes. En el caso de la producción de los investigadores de las universidades evaluadas en el presente estudio, casi en las tres cuartas partes de los artículos 
(71\%-73\%), publicaron como autor principal, como autor de correspondencia o ambos.

\section{Conclusiones}

Aunque han transcurrido 28 años de haberse iniciado el primer programa de posgrado en el área de las ciencias de la cultura física en México, es realmente muy poca la producción científica en el tema.

Existe una gran diferencia de producción entre las diversas instituciones educativas mexicanas ya que solo seis universidades son las que publican la mayoría de los artículos.

Los investigadores mexicanos no han incursionado en la publicación en revistas de mayor visibilidad y que sean específicamente del área que cultivan, las ciencias de la cultura física.

\section{Limitaciones del estudio}

Aun cuando la base de datos Scopus es la de mayor cobertura y nos proporciona una idea general muy cercana a la realidad, es de todas maneras una limitante, ya que existen más artículos publicados por investigadores mexicanos en esta área, en revistas que no están indizadas en esta base de datos, por lo que no se puede asumir que es toda la producción en ese campo de estudio.

\section{Referencias}

Acosta Ramírez, D. S., Ordaz Cortes, A. \& Baquero Parra, R. (2015) Análisis del desarrollo científico en la península de Yucatán: el punto de vista de los investigadores. IJERI: International Journal of Educational Research and Innovation, (4), 111-122.

Alonso Arroyo, A., Oliveira, E. F. T. D., Grácio, M. C. C., Pandiella, A. \& Aleixandre Benavent, R. (2016). Un análisis bibliométrico en el área de la Medicina: colaboración científica entre Brasil y España (2002-2011). Investigación bibliotecológica, 30(69), 205-230.

Arechavala Vargas, R. (2011). Las universidades y el desarrollo de la investigación científica y tecnológica en México: una agenda de investigación. Revista de la Educación Superior, 40(158), 41-57.

Cabrero-Mendoza, E. (2015). Principales logros y desafíos del Sistema Nacional de Investigadores de México a 30 años de su creación. CTS: Revista Iberoamericana de Ciencia, Tecnología y Sociedad, 10(28), 181-191.

Codina, L. (2018). Revisiones sistematizadas para trabajos académicos https://www.lluiscodina.com/revisiones-sistematizadas-fundamentos/ (consultado el 20 de mayo de 2018)

De Jesús Nascimento, M. (2011). Usuário da informação como produção científica e disciplina curricular: origem dos estudos e o ensino no Brasil. RDBCI: Revista Digital de Biblioteconomia e Ciência da Informação, 8(2), 41-71.

De Souza Mendes, M. I. B., da Silva, N. S. L., Ferreira, M. S., Dias, M.A., Palma, A., do Espírito-Santo, G. \& Chaves, P. N. (2017). Em pauta a produçãodo grupo de trabalho temático Activdade Física e saúde do Colegio Brasileiro de Ciências do e Esporte (1997-2011). Revista Basileira de Ciências do Esporte, 39(1), 17-23.

Devís-Devís, J., Valenciano-Valcárcel, J., Villamón, M., \& Samaniego, V. P. (2010). Disciplinas y temas de estudio en las ciencias de la actividad física y el deporte. Revista Internacional de Medicina y Ciencias de la Actividad Física y del Deporte, 10(37), 150-166

De Vries, W., González, G., León, P. \& Hernández, I. (2008). Políticas públicas y desempeño académico, o cómo el tamaño sí importa. CPU-e, Revista de Investigación Educativa, 7. Recuperado el [5 de octubre de2018], de http://www.uv.mx/cpue/num7/ inves/de vries politicas publicas.html
Hernández González, V., Reverter-Masià, J. \& Jove-Deltell, C. (2017). Scientific production of the professors of physical education and sport in Catalonia (2005-2009 versus 2010-2014). Sport TKRevista Euroamericana de Ciencias del Deporte, 6(1), 17-24.

Maranto Rivera, M. (2017). Incentivos, restricciones y tensiones de los académicos universitarios en Hidalgo: El caso del perfil deseable del programa de mejoramiento del profesorado en la Universidad Autónoma del Estado de Hidalgo. Tesis de Maestría. UAEH.

Marziale, M. H. P. (2011). Indicators of Ibero-American scientific production. Revista latino-americana de enfermagem, 19(4), $853-854$

Mattar, S. \& González, T. M. (2011). ¿Quién puede ser autor de un artículo científico? ¿ Yo, tú, el, ellos, nosotros?¿ Quién sabe?. Revista MVZ, (16).

Molina, P., Martínez-Baena, A., \& Villamón, M. (2017). Pedagogía de la Educación Física: análisis de las revistas españolas más productivas (2005-2014). Retos, Nuevas Tendencias en Educación Física, Deporte y Recreación, (31), 14-18.

Moreno-Doña, A., Arévalo, S. T., \& Gómez-Gonzalvo, F. (2020). Formación inicial de maestros de educación física: conectando un quehacer pedagógico decolonial con la intervención social, política e insurgente del espacio público. Retos, Nuevas Tendencias en Educación Física, Deporte y Recreación, 37(37), 605612 .

Ortega Toro, E. \& Salado, J. (2018). La calidad de las revistas científicas para el profesorado universitario de ciencias del deporte. BiD: textos universitaris de biblioteconomia i documentació, 40

Pérez-Gutiérrez, M., Lagos-Hernández, R. I. \& Izquierdo-Macón, E. (2016). Sport sciences'scientific production published in Chile (1912-2014): a bibliometric approach. Movimento, 22(4).

Piedra-Salomón, Y. \& Martínez-Rodríguez, A. (2007). Producción Científica. Ciencias de la Información, 38(3), 33-38d.

Ramírez, C., Reyna, M., García, A., Ortiz, X. \& Valdez, P. (2011). Formación científica de los egresados de tres programas de maestría en ciencias: seguimiento a 10 años. (1999-2009). Revista de la Educación Superior, 40(158), 91-103.

Reverter Masià, J., Hernández González, V., Jové Deltell, M. C., \& Legaz Arrese, A. (2013). Indicadores de producción de los profesores de Educación Física y Didáctica de la Expresión Corporal en España en la Web of Science. Perspectivas em Ciência da Informação, 18(3), 3-23.

Reverter-Masià, J., Hernández-González, V., Jove-Deltell, C., Fonseca, T. \& Legaz-Arrese, A. (2014). Papers production in the database web of science and scopus about physical education: comparative study between Spain and Brazil. Transinformação, 26(2), 113-124.

Reverter-Masià, J., Hernández-González, V., Jové-Deltell, C. \& LegazArrese, A. (2016). Producción en Web Of Science y Scopus de profesores funcionarios con sexenio de las ciencias del deporte en España. Revista Interamericana de Bibliotecología, 39(2), 149-162.

Salazar, C. M., Lozano, R. J., Tamara, R., Valencia, M. \& Lozano, E. G. M. Otra mirada al cuerpo en movimiento. experiencias de abordaje metodológico en la investigación de las ciencias del deporte y la actividad física. http://leecolima.net/agora/ca/c2/ pdf/95.pdf (consultado el 6 de agosto de 2018)

Subsecretía de Educación Superior https://www.ses.sep.gob.mx (consultado el 20 de junio de 2018)

Valenciano-Valcárcel, J., Devís-Devís, J., Villamón, M. \& Peiró-Velert, C. (2010). La colaboración científica en el campo de las ciencias de la actividad física y el deporte en España. Revista Española de Documentación Científica, 33(1). 90-105.

Valdez Ramírez, P. (2005). La enseñanza de la ciencia en México. Ingenierías, 8(26), 3-5.

Villamón-Herrera, M., Devís-Devís, J., Valencia-Peris, A. \& Valenciano-Valcárcel, J. (2007). Características y difusión de las revistas científico-técnicas españolas de ciencias de la actividad física y el deporte. El profesional de la información, 16(6), 605-615.

Zueck-Enriquez, MA., Chávez-Guerrero, A. \& Blanco-Vega, H. (2009). Facultad de Educación Física y Ciencias del Deporte: Historia y Reforma Curricular. Colección Textos Universitarios. Universidad Autónoma de Chihuahua. 